\title{
Performance analysis of OFDM for 4G wireless systems under various fading channels
}

\author{
Hema Thota ${ }^{1}$, Ch.Santhi Rani ${ }^{2}$, Kiranmayee Kagitha ${ }^{3}$,Anusha Balagam ${ }^{4}$, Anusha Goriparthi ${ }^{5}$,Naga Lakshmi Devarapalli ${ }^{6}$ \\ B.Tech, Electronics and Communication Engineering, DMS SVH College of Engineering, Machilipatnam, India ${ }^{1,3,4,5,6}$ \\ Professor, Electronics and Communication Engineering, DMS SVH College of Engineering, Machilipatnam, India ${ }^{2}$
}

\begin{abstract}
The era of entirely wireless, mobile communication is rapidly approaching.Increasingly; users are demanding versatile and convenient modes of communication along with high-speed broadband and technology in the premise of guaranteeing certain quality of communication without losing performance and efficiency. The challenges are to give users high-speed, high-performance in different channel conditions. So, Orthogonal Frequency Division multiplexing (OFDM) has been recognized as one of the most promising techniques to meet these challenges. Space Time block Coding (STBC) has drawn much attention in 4G wireless technologies just because of its decoding simplicity. This paper presents the performance evaluation of STBC-OFDM systems covering channel model and coding scheme, it includes Rayleigh fading channels by which it is observed that Bit Error Rate(BER) is reduced to achieve the high data rate.In this work, a performance criterion such as bit error rates for low SNR is analyzed. The BER for OFDM system over Rician fading channel is observed to be 0.1273.It has been reduced to 0.03919 over Rayleigh fading channel with Line of Sight (LOS) for the same Signal to noise ratio (SNR).
\end{abstract}

Keywords: Wireless Systems, OFDM, BER, STBC, MIMO, SNR.

\section{INTRODUCTION}

In a world of fast changing technology, there is a rising requirement for people to communicate and get connected with each other to have appropriate and timely access to information regardless of the location of the each individual. The increasing demands and requirements for wireless communication systems ubiquity have led to the need for the better fundamental issues in communication theory and their implications for the design of highlycapable wireless systems. The key challenge of future wireless communication systems is to provide high data rate wireless access at high quality of service. Since the availability of radio spectrum is limited and communication capacity needs cannot be met without a significant increase in communication spectral efficiency. The propagation conditions are hostile due to fading caused by destructive addition of multi-path components and interference from other users. Thus, it is required to radically increase spectral efficiency and to improve link reliability as a solution which can be achieved by MIMO wireless technology.

Multiple-Input Multiple-Output (MIMO) wireless technology seems to meet these demands by offering increased spectral efficiency. The MIMO system spreads the total transmitted power over the antennas which give us more spectral efficiency and link reliability which reduced fading without increasing transmitted power or bandwidth. Thus, high data throughput and link range can be achieved. MIMO technology plays an important role in modern wireless communication standards such as Long Term Evolution (LTE) developed by $3^{\text {rd }}$ Generation Partnership Project (3GPP), 4G due to having these properties.

This paper is organized into five sections where in section I give introduction and MIMO-OFDM approach is dealt with in section II. Section III gives about the Space Time Block Coding and channel model that has been taken into consideration while results and discussion and conclusions are made in sections IV and V respectively.

\section{A. Literature Survey}

Although, the studies and system based on the concept of orthogonal frequency division multiple access have been publishing since 1958, the most of the application using current form of OFDMA developed during 1980s and 90s. There is a lot of research on increasing the data rate. Whereas this paper discusses how the BER can be reduces over various Fading channels.

The authors in [1] evaluated the performance of IEEE 802.16- 2004 and they found that the rate of data transmission is being increased.

The researchers in [3] analyzed the performance of two transmit antennas with more receive antennas is much better than that of the system with two transmit antenna and less receive antennas in term of BER due to the more diversity gain of Alamouti's code.

\section{MIMO - OFDM SYSTEM}

OFDM is an efficient technique for transmitting data over frequency selective channels. The main idea behind OFDM is to divide a broadband frequency channel in to a few narrowband sub-channels. Then, each sub-channel is a flat fading channel despite the frequency selective nature of the broadband channel. To generate these parallel subcarriers in OFDM, an Inverse Fast Fourier transform (IFFT) is applied to a block of $L$ data symbols. To avoid ISI due to the channel delay spread, a few "Cyclic Prefix" (CP) symbols are inserted in the block. The cyclic prefix samples are also called guard intervals. Basically, the last $g$ samples of the block are duplicated in front of the block as the cyclic prefix. The number of these cyclic prefix samples, should be bigger than the length of the channel 
impulse response. The effects of the cyclic prefix samples eliminate ISI and convert the convolution between the transmit symbols and the channel to a circular convolution. These cyclic prefix samples are removed at the receiver. Then, a Fast Fourier Transform (FFT) is utilized at the receiver to recover the block of $L$ received symbols. The Fig. 1 shows block diagram of a wireless communication system using OFDM over a SISO channel. This paper presents OFDM system designed for MIMO channels. This is usually called MIMO-OFDM.

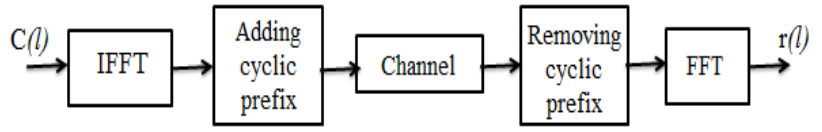

Fig.1 Block diagram of SISO- OFDM system.

\section{STBC FOR OFDM SYSTEM AND CHANNEL MODELS}

\section{A. $\quad$ Space Time Block Codes}

In this paper, space-time block codes are presented and the performance of MIMO fading channels is evaluated by Simulations. The Alamouti code is one of the block codes, which is a simple two branch transmit diversity scheme. The key features of the scheme is orthogonally between the sequences generated by two transmit antennas.

The space-time block codes can achieve the full transmit diversity specified by the number of the transmit antennas $\mathrm{n}_{\mathrm{T}}$, while allowing a very simple maximum-likelihood decoding algorithm, based only on linear processing of the received signals.

The Fig.2 shows an encoder structure for STBC. In general, a space-time block code is defined by a $n_{T X P}$ transmission matrix $\mathrm{X}$. Here $\mathrm{n}_{\mathrm{T}}$ represents the number of transmit antennas and $\mathrm{p}$ represents the number of time periods for transmission of one block of coded symbols.

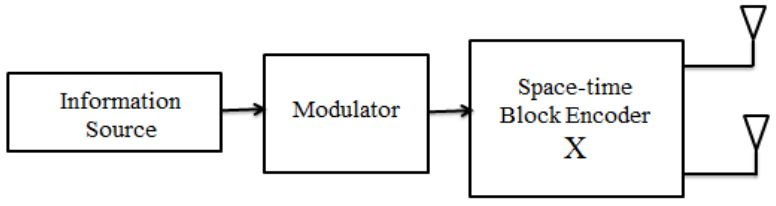

Fig. 2 Encoder for STBC

\section{B. $\quad$ Space Time Block Code used in OFDM System} The Space Time Block codes are used in MIMO-OFDM because of its decoding simplicity. In this paper, two transmit antennas and one receive antenna is considered. Fig.3 (a) and (b) shows the transmitter \& Receiver of STBC-OFDM System including Space Time Block encoder and decoder.

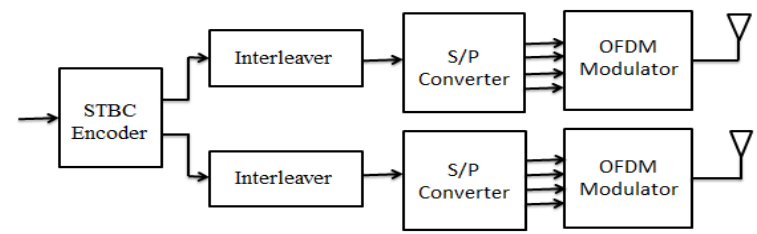

(a) Transmitter

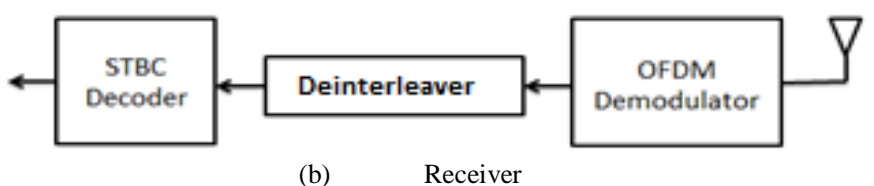

(b)

Receiver

Fig.3 An STBC-OFDM system block diagram

\section{MIMO Channel Model}

In wireless communications, multipath propagation occurs because of reflection, diffraction, scattering and shadowing of the transmitted signals due to surrounding objects as a result the transmitted signals arrive at the receiver with different amplitude, different phase angles and at different time intervals. The amplitude fluctuation of the received signal is called signal fading and we assume the fading process follows a Rayleigh probability distribution function. In time domain, the channel response from the $i^{\text {th }}$ Transmit antenna to the $j^{\text {th }}$ receive antenna can be given as

$$
h_{i, j}(t)=\sum_{l=0}^{L-1} \alpha_{i, j}(l) \delta\left(t-\tau_{l}\right)
$$

Where $\alpha_{i, j}(l)$ is the multi-path gain coefficient, $\mathrm{L}$ denotes the number of resolvable paths, and $\tau_{l}$ represents the path delay time of $l^{\text {th }}$ multi-path component. The frequency response of the channel is given by

$$
h_{i, j}^{k}(f)=\sum_{l=0}^{L-1} \alpha_{i, j}^{k}(l) e^{-j 2 \pi f \tau_{l}}
$$

Since, two transmit antennas and one receive antenna are considered, the values of $i$ are 1,2 and, $\mathrm{j}$ is 1 .

\section{Statistical Models for Fading Channels}

Because of the multiplicity of factors involved in propagation in a cellular mobile environment, it is convenient to apply statistical techniques to describe signal variations. In a narrowband system, the transmitted signals usually occupy a bandwidth smaller than the channel's coherence bandwidth, which is defined as the frequency range over which the channel fading process is correlated. That is, all spectral components of the transmitted signal are subject to the same fading attenuation. This type of fading is referred to as frequency nonselective or frequency flat. In this section Rayleigh and Rician fading models are introduced to describe signal variations in a narrowband multipath environment.

1) Rayleigh Fading: The transmission of a single tone with constant amplitude is considered. In a typical land mobile radio channel, it is assumed that the direct wave is obstructed and the mobile unit receives only reflected waves. When the number of reflected waves is large, according to the central limit theorem, two quadrature components of the received signal are uncorrelated Gaussian random processes with a zero mean and variance $\sigma_{s}^{2}$. As a result, the envelope of the received 
signal at any time instant undergoes a Rayleigh probability distribution and its phase obeys a uniform distribution between $-\pi$ and $\pi$. The probability density function (PDF) of the Rayleigh distribution is given by

$$
p(a)=\left\{\begin{array}{cc}
\frac{a}{\sigma_{s}^{2}} \cdot e^{-a^{2} / 2 \sigma_{s}^{2}} & a \geq 0 \\
0 & a<0
\end{array}\right.
$$

2) Rician Fading: In some propagation scenarios, such as satellite or microcellular mobile radio channels, there are essentially no obstacles on the line-of-sight path. The received signal consists of a direct wave and a number of reflected waves. The direct wave is a stationary nonfading signal with constant amplitude. The reflected waves are independent random signals. Their sum is called the scattered component of the received signal. The sum of a constant amplitude direct signal and a Rayleigh distributed scattered signal results in a signal with a Rician envelope distribution. The PDF of the Rician distribution is given by

$$
\begin{aligned}
& p(a) \\
& = \begin{cases}\frac{a}{\sigma_{s}^{2}} \cdot e^{\frac{-\left(a^{2}+b^{2}\right)}{2 \sigma_{s}^{2}}} I_{0}\left(\frac{a D}{\sigma_{s}^{2}}\right) & a \geq 0 \\
0 & a<0\end{cases}
\end{aligned}
$$

\section{Simulation Results}

Simulation results are presented to evaluate the performance of OFDM system with two transmit antennas and one receive antenna. Fig.4 shows the performance analysis of OFDM system with STBC on Rician channel and performance analysis of OFDM system with STBC under Rayleigh fading channel with LOS is shown in the Fig.5.

\section{A. Performance Analysis of OFDM system with} STBC under Rician channel

In the Fig.4, simulation results for the performance of OFDM system without STBC under Rician fading channel are shown. The performance of the system is analyzed with various low SNR values ranging from $0 \mathrm{~dB}$ to $20 \mathrm{~dB}$.

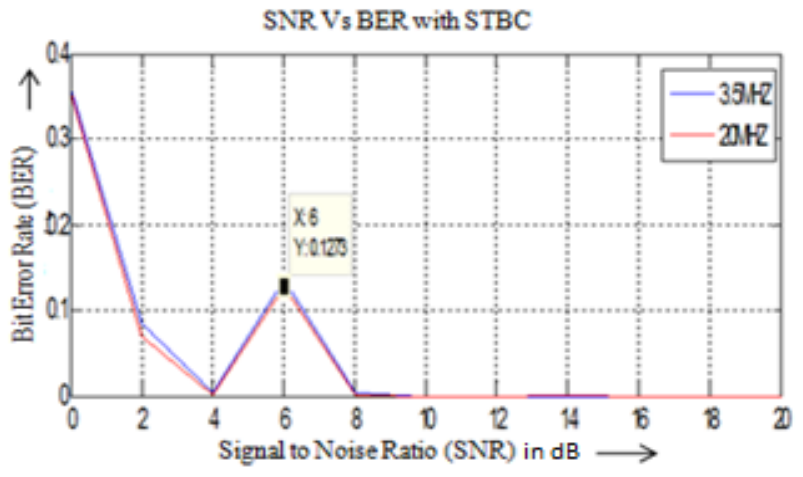

Fig.4.BER performance of OFDM System with STBC under Rician channel
The Simulation model employs various modulation schemes based on Rate_Id, indicating the modulation technique used and the modulation rate. the values of Rate_Id are 0 for BPSK1/2,1 for QPSK1/2,2 for QPSK3/4,3 for 16-QAM1/2,4 for 16-QAM3/4,5 for 64QAM2/3 and 6 for 64-QAM3/4.

In Fig.4, it is observed that the value of BER at SNR of $6 \mathrm{~dB}$ is 0.1273 for the channel bandwidth of $3.5 \mathrm{MHZ}$ and 20MHZ

B. $\quad$ Performance Analysis of OFDM system with STBC under Rayleigh fading channel

In this fig.5, simulation results for the performance of STBC on Rayleigh fading Channels with low SNR values ranging from $0 \mathrm{~dB}$ to $20 \mathrm{~dB}$ are shown. It is observed that the value of BER at SNR of $6 \mathrm{~dB}$ is 0.03919 for the channel bandwidth of $20 \mathrm{MHZ}$ and 0 for $3.5 \mathrm{MHZ}$.

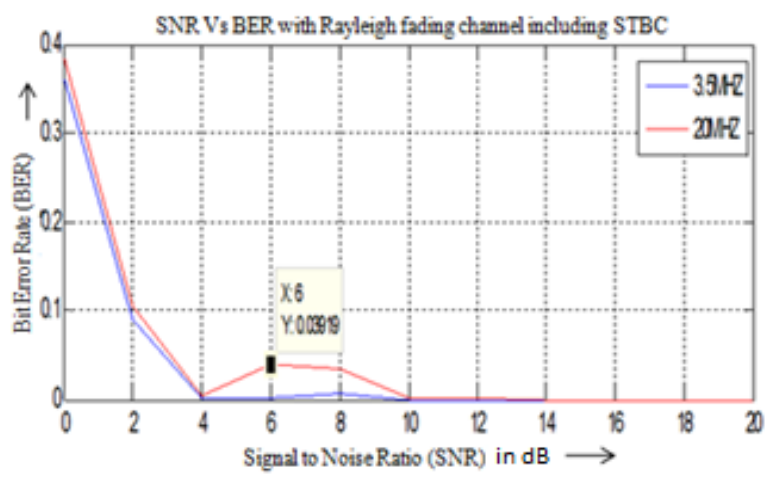

Fig.5. BER performance of OFDM System with STBC under Rayleigh fading channel

In both the simulation results provided above, we observe that in fig. 4 the value of BER is 0.1273 for SNR of $6 \mathrm{~dB}$ whereas in fig. 5 the value of BER reduces to 0.03919 for same SNR. Thus reduction of BER imposes that number of bits that are being transmitted increased, indicating high bit rate. Hence, performance of OFDM system increases under Rayleigh fading channel than the performance under Rician fading channel alone.

\section{Conclusion}

In this paper, the performance of the MIMO-OFDM system under various fading channels is evaluated. It is observed that for low SNR values ranging from $0 \mathrm{~dB}$ to $20 \mathrm{~dB}$, the BER for OFDM system over Rician fading channel is 0.1273 and is reduced to 0.03919 over Rayleigh fading channel and LOS for the signal to noise ratio(SNR) of $6 \mathrm{~dB}$ Rate. As BER is reduced, the number of bits transmitted to receiver are increased which implies that high bit rate is achieved.

\section{ACKNOWLEDGEMENT}

We take immense pleasure to express our profound gratitude and heartfelt thanks to Dr.Ch.Santhi Rani, Professor, ECE for her exemplary guidance, monitoring and constant encouragement throughout the completion of our final year B.Tech project. The blessing, help and guidance given by her from time to time shall carry us a long way in the journey of life on which we are about to embark. 
We also express a deep sense of gratitude to thank Dr.K.V.S.V.R.Prasad, Head of the Department, ECE and Dr.K.SuryaPrakasaRao, Principal, DaitaMadhusudhanaSastry Sri Venkateswara Hindu college of Engineering, Machilipatnam for providing well organized infrastructure being helpful in completion of our project.

\section{REFERENCES}

[1] Hussain A. Alhassan, Dr. Eman Abdel Fattah, "A Study on the Performance of IEEE 802.16-2004 Includes STBC" ASEE 2014 Zone I Conference, April 3-5, 2014, University of Bridgeport, Bridgpeort, CT, USA

[2] G. J. Foschini and M. Gans, "On the limits of wireless communication in a fading environment when using multiple antennas", Wireless Personal Communication, vol. 6, pp. 311-335, Mar. 1998.

[3] Md. Mejbaul Haque1,*, Mohammad Shaifur Rahman1 and Ki-Doo Kim2,"Performance Analysis of MIMO-OFDM for 4G Wireless Systems under Rayleigh Fading Channel" International Journal of Multimedia and Ubiquitous Engineering Vol. 8, No. 1, January, 2013

[4] J. Yuan, Z. Chen, B. Vucetic and W. Firmanto, "Performance analysis of space-time coding on fading channels", in Proc. IEEE ISIT'01, Washington D.C., pp. 153, Jun. 2001.

[5] J. Yuan, Z. Chen, B. Vucetic and W. Firmanto, "Performance analysis and design of space-time coding on fading channels", submitted to IEEE Trans. Commun..

[6] S. M. Alamouti, "A simple transmit diversity technique for wireless communications", IEEE Journal Select. Areas Commun., vol. 16, no. 8, pp. 1451-1458, Oct. 1998 .

[7] Lu, B. and Wang, X. Space-time code design in OFDM systems. IEEE Globecom, 2: Nov. 2000, 1000-4.

[8] Molisch, A. F., Win, M. Z. and Winters, J. H. Space-time-frequency (STF) coding for MIMO-OFDM systems. IEEE Communications Letters, 6(9): Sept. 2002, 370-2.

[9] Lu, H.-F., Kumar, P. V. and Chung, H. On orthogonal designs and space-time codes.IEEE International Symposium on Information Theory (ISIT), June 2002, 418.

[10] Tarokh, V., Jafarkhani, H. and Calderbank, A. R. Space-time block codes from orthogonal designs. IEEE Trans. on Information Theory, 45(5): July 1999, 1456-67.

[11] Tarokh, V., Naguib, A., Seshadri, N. and Calderbank,” A. R. Spacetime codes forhigh data rates wireless communications: performance criteria in the presence of channel estimation errors, mobility and multiple paths" IEEE Trans. On Communications, 47(2): Feb. 1999, 199-207.

$$
\text { P.Sunil }
$$

Ms.M.Sarumathi,"Performance Comparison of Rayleigh and Rician Fading Channels In QAM modulation Scheme Using Simulink Environment'International Journal of Computational Engineering Research\|Vol, 03\|Issue, 5\| 